\title{
Las segundas partes sí pueden ser mejores: Algunas Reflexiones sobre el uso de datos secundarios en la investigación cualitativa
}

\section{Resumen}

El análisis de datos secundarios es una de las estrategias más "antiguas" que los sociólogos han empleado para dar cuenta de fenómenos de la realidad social sobre los que se disponía de información elaborada por otros

El presente trabajo tiene por objetivo establecer una reflexión sistemática sobre el uso de información secundaria en los procesos de investigación social cualitativos.

El articulo consta de cuatro partes: 1.- hacia una definición de dato y análisis secundario; 2. ventajas y desventajas del uso de información secundaria; 3.cómo, por qué y para qué re-usar información cualitativa, y 4.- algunas propuestas a modo de apertura de un campo de discusión.

Abogamos por establecer criterios claros que puedan facilitar el uso y análisis de información elaborada por otros y/o en diferentes contextos de producción.

Palabras clave: Datos secundarios. Investigación cualitativa. Re-uso de información.

1 Investigador del CO NICET. CEA-U NIDAD EJECUTORA-UNC. Coordinador del Programa de Estudios sobre Acción Colectiva y Conflicto Social; Coordinador del Grupo de Estudio sobre Sociología de las Emociones y el Cuerpo. Instituto Gino Germani. UBA; Profesor del IAPCS-U NVM

2 Docente en la U niversidad de Buenos Aires. M iembro del Grupo de Estudios de M etodología del Instituto de Investigaciones Gino Germani. Facultad de Ciencias Sociales. U BA.(GEM IS) 


\section{Introduccion}

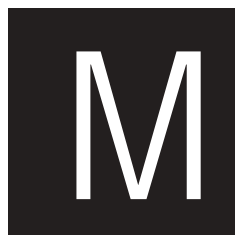

uchas vecesal comentar con amigosel próximo estreno de la segunda parte de una película, aparece con mucha frecuencia la expresión "... las segundas partes nunca son buenas...". En la investigación social no hay segundas partespero muchas veces usamos información que otros han producido con anterioridad o que nosotros mismos hemos elaborado en el pasado.

Un sinnúmero de preguntas suelen hacerse sobre la validez, la comparabilidad, y la inscripción teórica de informaciones que no han sido construidas con los mismos propósitos, con la misma estrategia de indagación y desde diferentes perspectivas teóricas. En este contexto, las más de las veces respecto a estrategias cuantitativas, se plantean las reflexiones sobre el uso y administración de "datos secundarios".

El presente trabajo tiene por objetivo establecer una reflexión sistemática sobre el uso de información secundaria en los procesos de investigación social cualitativos. Para alcanzar dicho objetivo se ha seguido la siguiente estrategia expositiva: 1) se hace una reseña de algunas maneras de definir a los "datos secundarios" tanto en estrategias cuantitativas como cualitativas; 2) se sintetiza las potencialidades y obturaciones que emergen del uso de información secundaria; 3) se propone y justifica la utilización de información secundaria en los diseños cualitativos; y 4) se concluye con algunas propuestas para potenciar y mejorar dicha utilización. Se termina abogando por avanzar en procesos colaborativos de investigación con la articulación de diversasfuentes de información.

\section{Hacia una definicion de dato y analisis secundario}

El análisis de datossecundarios es una de las estrategias más "antiguas" que los sociólogos han empleado para dar cuenta de fenómenos de la 
realidad social sobre los que se disponía de información elaborada por otros. Dos ejemplos claros lo constituyen Durkheim y Marx.

En los "M anuales de M etodología”, más usados, la problemática suele inscribirse en el desarrollo de la temática sobre el uso de fuentes de información secundaria y/o aparece asociada a la selección del tema/ planteamiento del problema ${ }^{3}$.

En, -un no tan breve- recorrido por distintos manuales de metodología es posible observar por un lado la falta de mención a las investigaciones con datos secundarios (por ejemplo Padua 2000; Mayntz 2004; Galtung; 1978), por otro la mención (tal vez escueta) específicamente ligada a investigaciones de tipo cuantitativas (por ejemplo Sautu et alt 2005; Grawitz 1975).

Entre las distintas formas de definir este tipo de estrategia de investigación hallamos un modo contundente expuesto por Sierra Bravo:

Se entiende por análisis secundario de documentos o de datos documentales todo posterior análisis de un conjunto de datosprimarios que ofrezca interpretaciones y conclusiones adicionales o en forma diferente a la presentada en el primer informe de investigación. (SIERRA BRAVO, 2003, p. 292)

Siguiendo al mismo autor, ello implica dos requisitos: 1) un análisis posterior o re análisis de datos obtenidos y analizados anteriormente; 2) que el nuevo análisis no sea repetición de los análisis conocidos ya realizados con los datos, sino hacer tratamientos diferentes, interpretaciones distintas, adicionales.

En todos los casos, los diversos autores resaltan la importancia de la validez y fiabilidad de la información, es decir considerar fuertemente aspectos teóricos-epistemológicos y metodológicos tales como, el proceso de captación de la información; las definiciones utilizadas al recogerse la 
información; la formación del investigador respecto al contexto en que se relevo la información. Rojas Soriano advierte que cuando se utiliza información secundaria, el investigador debe preguntarse ¿Q ué validez tiene para el problema que se estudia? ¿La perspectiva teórica (y la ideología subyacente) con que se obtuvo la información, corresponde a la utilizada en la propia investigación? Sin olvidar que la importancia de la construcción del dato, supone una adecuada reflexión crítica. Remarcando que un objeto de investigación es definido y construido en función de una problemática teórica.

Según Selltiz (1980), los datos secundarios sirven también para la formulación de hipótesis de investigación; pero para utilizarlos se necesita de una capacidad para formular muchas y distintas preguntas relacionadas con el problema de investigación. El principio básico por tanto, para el uso de estadísticas disponibles consiste en ser flexible con respecto a la forma en que se formulan las preguntas de la investigación (SELLITZ, 1980, p. 510)

En los últimos 10 años en la sociología que opta por estrategias cualitativas de indagación las discusiones sobre el valor, sentido y procedimientos para realizar análisis de datos secundarios se han vuelto usuales. ${ }^{4}$

En esta dirección refiriéndose específicamente a las indagaciones cualitativas, Mruck destaca la necesidad de discutir más detenidamente las implicaciones metodológicas del análisis secundario; en este caso señala cuestiones sobre la anonimización, confidencialidad y ética como a medios válidos y creativos de ahorrar recursos para formular "nuevas preguntas a viejos datos".

En su artículo "Secondary analysis of qualitative data" Janet Heaton sostiene;

El análisis secundario implica la utilización de datos existentes, recogidos a los efectos de un estudio previo,

4 Para mencionar tres experiencias claras de dichas discusiones se pueden ver las publicaciones de carácter internacional FQ S: Forum: Q ualitative Social Research; IJSRM : International Journal of Social Research M ethodology, MI: M ethodological Innovations O nline 
con el fin de usarlos en una investigación de un interés que es distinto al de la indagación original, que podrá (involucrar) una nueva pregunta de investigación o una perspectiva alternativa sobre la pregunta original. (HEATO N, 1998)

Goodwin y 0 'Connor en un trabajo orientado a justificar los beneficios de utilizar las notas de campo de los entrevistadores originales al usar datos cualitativos producidos por otros afirman;

Con el análisis secundario de los datos cualitativos se abre la posibilidad de explorar temasy debates actuales a través de los datos que se recogieron en el pasado. (GOODWIN ; O'CONNOR, 2006)

Kelder por su parte ha señalado respecto al análisis secundario de datos cualitativos:

Los datos cualitativos son capaces de ser revisados desde múltiples perspectivas, y se utilizan para responder a preguntas de investigación diferentes a las previstas por el investigador que obtuvo los datos originales. El uso de datos de otras personas ahorra tiempo, evita sobrecargar innecesariamente a los participantes de la (propia) investigación, y agrega confianza a la interpretación de los propios datos. (KELDER, 2005)

A la luz de lo expuesto se hace evidente una de las líneas maestras de la metodología: los datos no hablan por sí mismos. La potencialidad implícita de toda construcción de información refiere a la posibilidad de "mirarla" de diferente manera, desde odres teóricos, experienciales (y por que no políticos) diversos a los que utilizara su constructor.

La utilización de información recabada no por el propio investigador, sino por otro/as autores, refiere al uso y -en algunoscasos- el procesamiento de bases de información que diversos investigadores, organismos públicos 
o privados realizan con objetivos propios concernientes a una investigación o relevamiento, entre los que se encuentran -al menos- los archivos documentales y estadísticos. Dicha modalidad, utilizada como única -0 complementando otras- se encuadra dentro de las denominadas investigaciones con fuentes secundarias y marcadas por el hecho que el investigador no es quien acude a la búsqueda de la información.

Es menester considerar que, los objetivos para los cuales se recogió la información son distintos de aquellos para los que se desea utilizar como fuente secundaria, por ello requiere una tarea adicional para el investigador, como es familiarizarse con dicha fuente y su construcción. Luego de ello, efectuará los análisis de acuerdo a los propósitos planteados reintegrando la información o reprocesándola.

Preliminarmente digamosque se puede entender por "dato secundario" al registro de una observación realizada (por el mismo u otro investigador) en contextos de producción diferentesa los cuales se los pretende inscribir en una oportunidad determinada. Con el mismo carácter es posible entender al "análisis secundario de datos cualitativos", como el procedimiento mediante el cual un investigador utiliza información registrada por otros reconstruyendo su descripción y sistematización desde una estrategia de indagación diferente a la original 5 .

Ahora bien, sea en estrategias cuantitativas como en cualitativas el uso de información elaborada por otro o por el propio investigador en otro contexto de producción, como ya adelantáramos, conlleva ventajas y desventajas, repasemos ahora alguna manera de esquematizarlas.

\section{Ventajas y desventajas del uso de informacion secundaria}

Cuando un investigador se decide por usar información construida por otro u en otros contextos de indagación emergen varias preguntas: ¿Un

5 Scribano ha expuesto de manera extensa la noción de "dato cualitativo", análisise interpretación de losmismos en Scribano 2008 
conjunto de "datos" no depende de las preguntas de investigación desde las cuales se hayan construido? ¿Los esquemas teóricos no influyen de manera terminante en la elaboración de informaciones volviendo a estas "dependientes" de los mismos? ¿Los contextos de obtención de la información no "marcan" enfáticamente a la misma? ¿Lossujetoscon loscualesse ha compartido la indagación y que son los que han "cedido" la información avalarían su re-uso? Responder a cada una de estas preguntas de manera exhaustiva demandaría la elaboración de otro trabajo paralelo al presente. Lo que sí podemos-y queremos- hacer aquí es dejar constancia de loslímites y posibilidades del análisis secundario de modo tal que quede allanado el camino para discutir su importancia. Desde una perspectiva global, el análisis de datos secundarios, presenta una serie de ventajas y desventajas, que pueden sintetizarse del siguiente modo:

Tabla 1. Ventajas y desventajas del analisis secundario

\begin{tabular}{|l|l|}
\hline \multicolumn{1}{|c|}{ Ventajas } & \multicolumn{1}{|c|}{ Desventajas } \\
\hline - Minimiza los efectos que la presencia & - El investigador debe comprobar la validez \\
del investigador puede ocasionar en la & y fiabilidad de la fuente. \\
obtención de la información. & El investigador debe revisar: la \\
- Más económica en tiempo y en recursos & consistencia de la información; el diseño \\
humanos y materiales. & muestral; la técnica de obtención de la \\
- Facilita el análisis comparativo y el de & $\begin{array}{l}\text { información; la cantidad de respuestas } \\
\text { obtenidas; la fecha de obtención de la }\end{array}$ \\
tendencias. & $\begin{array}{l}\text { - Posibilita la formulación de muchas y dis- } \\
\text { información: los conceptos utilizados y su } \\
\text { tintas preguntas relacionadas con el pro- } \\
\text { blema de investigación. }\end{array}$
\end{tabular}

En la investigación cualitativa existen hoy un sinnúmero de publicaciones que dan cuenta de las ventajas y desventajas de usar informaciones producidas con anterioridad en otros proyectos de investigación. Una manera de esquematizar dichas ventajas y desventajas es usando el tipo de fuente de la cual obtenemosla aludida información, un esquema posible es el que sigue: 
Tabla 2. Ventajas y desventajas del acceso a informacion secundaria cualitativa de acuerdo al tipo de fuente de las que se pueden obtener (elaboración propia en base a corti 2000)

\begin{tabular}{|c|c|c|c|}
\hline TIPO FUENTE & $\begin{array}{l}\text { INFORMACION A LA } \\
\text { QUESEACCEDE }\end{array}$ & VENTAJAS & DESVENTAJAS \\
\hline $\begin{array}{l}\text { ArchivosTradicionales, } \\
\text { Bibliotecas }\end{array}$ & $\begin{array}{l}\text { Documentospersonales } \\
\text { de académicosque } \\
\text { contienen los datos, } \\
\text { métodosy documentos, } \\
\text { informes sobre } \\
\text { estrategias de } \\
\text { indagación, e } \\
\text { investigación sustantiva, } \\
\text { correspondencia sobre el } \\
\text { diseño de investigación }\end{array}$ & $\begin{array}{l}\text { Fiabilidad Institucional } \\
\text { Acceso a material } \\
\text { "privado" de los } \\
\text { investigadores }\end{array}$ & $\begin{array}{l}\text { Difícil conexión con los } \\
\text { investigadores originales y casi } \\
\text { nula con lossujetos "investigados" }\end{array}$ \\
\hline $\begin{array}{l}\text { DocumentosO ficinales } \\
\text { y M useos }\end{array}$ & $\begin{array}{l}\text { Coleccionestemáticas } \\
\text { por ejemplo, de } \\
\text { colecciones locales de } \\
\text { entrevistas de la historia } \\
\text { oral }\end{array}$ & $\begin{array}{l}\text { Fiabilidad Institucional } \\
\text { Coherencia } \\
\text { Programática }\end{array}$ & $\begin{array}{l}\text { Difícil conexión con los } \\
\text { investigadores originales y muy } \\
\text { difícil con los sujetos "investigados" }\end{array}$ \\
\hline $\begin{array}{l}\text { Gruposde Investigación, } \\
\text { redesacadémicasque } \\
\text { participan en } \\
\text { investigaciones } \\
\text { cualitativas }\end{array}$ & $\begin{array}{l}\text { Diversas colecciones de } \\
\text { datos de proyectosde } \\
\text { investigación anteriores }\end{array}$ & $\begin{array}{l}\text { Coherencia } \\
\text { Programática } \\
\text { Posibilidad de } \\
\text { Contacto con el Grupo } \\
\text { de Investigación }\end{array}$ & $\begin{array}{l}\text { Difícil accesibilidad material de la } \\
\text { información }\end{array}$ \\
\hline $\begin{array}{l}\text { Investigadores } \\
\text { Individuales }\end{array}$ & $\begin{array}{l}\text { Colecciones personales, } \\
\text { a menudo en torno a } \\
\text { temas similares }\end{array}$ & $\begin{array}{l}\text { Coherencia } \\
\text { Programática } \\
\text { Posibilidad de } \\
\text { Contacto con el } \\
\text { investigador y } \\
\text { material conexo }\end{array}$ & $\begin{array}{l}\text { Difícil accesibilidad material de la } \\
\text { información } \\
\text { Disponibilidad del Investigador }\end{array}$ \\
\hline $\begin{array}{l}\text { Archivosy Bases de } \\
\text { Datos; Bibliotecas } \\
\text { Digitales }\end{array}$ & $\begin{array}{l}\text { Datos, notas de campo y } \\
\text { diseños de investigación }\end{array}$ & $\begin{array}{l}\text { Fiabilidad Institucional } \\
\text { Coherencia } \\
\text { Programática }\end{array}$ & $\begin{array}{l}\text { Difícil accesibilidad material de la } \\
\text { información } \\
\text { Disponibilidad del Investigador y o } \\
\text { Grupo de Investigación }\end{array}$ \\
\hline
\end{tabular}

En este marco, haciendo énfasis en el análisis de dato cualitativo secundario, y solo para simplificar nuestra exposición, hemos re-construido una lista breve de las ventajas y desventajas:

Ventajas:

1. Contar con información que se articule con la que dispone "primariamente" el investigador en orden a profundizar una mirada más adecuada del fenómeno que quiere estudiar.

2. Disponer de mayor "evidencia" para las afirmaciones que pretende sostener el investigador en su indagación. 
3. Co-construir más "participativamente" una visión del mundo social que implique una multiplicidad de voces cada vez mayor

Desventajas:

1. Las limitaciones éticas dados los consentimientos, objetivos e intereses de TODOS los sujetos que hayan participado en la investigación que da origen a la información por re-usar.

2. Las dificultades que implica las limitaciones de hacer reflexivos los contextos de observación, metodológicos, teóricos, epistémicos y políticos que ha tenido la investigación usada.

3. Las obturaciones de "re-transmisión" de los rasgos preceptúales y dialógales que toda investigación cualitativa implica.

Más allá de lo afirmado hasta aquí respecto a obstáculosy posibilidades el análisis de datos secundarios cualitativo no solo es posible sino que hasta deseable; en el próximo apartado damos algunas pistas de ello.

\section{Como, por qué y para qué} re-usar informacion cualitativa.

Usar y re-producir informaciones elaboradas en contextos de investigación diferentes al propio implica la aplicación constante de una vigilancia epistemológica que permita hacer reflexivos y apropiables ambos procesos de producción de conocimiento.

Las proximidad-distancia, la conexión-desconexión entre las indagaciones teniendo en cuenta las superficies de inscripción donde (y desde donde) se las signifique y los entramados teóricos que le dan "coherencia" posibilitan evaluar las posibilidades de éxito de su articulación.

Una vía para realizar la aludida evaluación puede ser la de concentrarse en: O bjetivos, Estrategia M etodológica, Posición(es) del/los N arrador(es) . 


\section{Tabla 3.}

\begin{tabular}{|l|l|l|l|}
\hline & O bjetivos & $\begin{array}{c}\text { Estrategia } \\
\text { M etodologica }\end{array}$ & $\begin{array}{c}\text { Posición(es) } \\
\text { Narrador(es) }\end{array}$ \\
\hline Idénticos & & & \\
\hline Similares & & & \\
\hline Diferentes & & & \\
\hline
\end{tabular}

Las "condiciones ideales" del uso de información cualitativa elaborada por otros se la obtiene cuando usando imaginariamente la Tabla 3 podemos tildar la celdas que cruzan la fila "Idénticos" con las tres columnas de la misma. Es decir, si los objetivos, la estrategia metodológica y la posición del narrador son las mismas en la investigación en curso y en aquella de la cual hemos obtenido la información a usar. Esta posibilidad no esinfrecuente si se piensa en Programas de Investigación compuestos por diversos proyectos de investigación que indagan (y construyen) objetos diferentes pero con problemas complementarios y la misma mirada teórica. Los ejemplos más evidentes son las relaciones entre Programas de Investigación y las Tesis elaboradas en esos contextos; y/o las proximidades entre Grupos de Investigación que conforman una Red de Estudios.

O bviamente, las condiciones de uso, (que podemos denominar preliminarmente como de "justificación dificultosas"), sobre las que hay que tener una actitud reflexiva de constante y mayor vigilancia epistemológica se las tiene cuando podemos tildar imaginariamente, en la Tabla aludida, las celdas que cruzan la fila "D iferentes" con todas las columnas. Esta posibilidad es muy frecuente y se puede comprender muy bien si se repara en la existencia (bienvenida por cierto) cada vez más extendida en America Latina de Archivos de H istoria O ral. Dichas historias han sido construidas con objetivos, estrategias metodológicas y con una posición de los narradores coherentes con los investigadores encargados del Archivo en cuestión y es por eso mismo que su uso, más allá de tener que pasar por una justificación adecuada, se vuelve más operativo. 
La situación intermedia de la fila "Similares" es más difícil de precisar pero, dada la interacción actual entre grupos de investigación, suele ser la más usual. En Congresos, a través de publicaciones periódicas, por estancia de investigación en otros lugares diferentes a los propios; el investigador Latinoamericano se encuentra hoy muy asiduamente con personas que, si bien "no trabajan lo mismo" lo hacen en algo muy similar, y que quieren (pueden) compartir información.

De la mucha información disponible hemos seleccionado tres tipos de recomendaciones, a) una que intenta esquematizar el cómo y el por qué de la conveniencia de hacer el análisis secundario de datos cualitativos, b) otra que adelanta una serie de ejes como guía para hacerlo, y c) finalmente otra preocupada en como "retomar" el contexto de la información secundaria.

a) En una interacción no muy bien definida entre el cómo y por qué usar datos secundarios Corti ha elaborado la siguiente lista:

- "nuevas preguntas para los viejos datos": abordar los datos de manera que no sea la dirección original. Cuanto más se llegue al fondo del material, más esto es posible,

- para el diseño de la investigación: la utilización del muestreo y técnicas de recolección de datos y herramientas para diseñar una nueva investigación, o el estudio para la metodología en sí,

- caso como material para la enseñanza: el uso de la metodología, datosy métodos, por ejemplo, los estudios clásicos para la enseñanza de métodos de investigación a través de una gama de disciplinas de las ciencias sociales,

- para la investigación comparativa: los datos se pueden comparar con otros datos nuevos (o fuentes), a través del tiempo o de la región, grupo social, etc,

- para la verificación: para fundamentar los resultados.

- históricamente: datoscreados ahora inevitablemente se convertirá en un recurso histórico." (CO RTI, 2000, p. 47) 
b) N otz haciendo reflexiva su propia práctica de análisis secundario, sugiere como "pasosa seguir": en primer lugar describir los datos que se van utilizar y en segundo lugar establecer criterios de uso de los contenidos de dichos datos. En cuanto a las informaciones surgidas de las entrevistas, el caso que le preocupa un Not, postula la posibilidad de "focalizarse en casos particulares" o "focalizarse en tópicos" seleccionados para el análisis. En ese contexto sostiene la necesidad de tener presente los siguientes elementos para realizar el análisis:

- "Antes de llevar a cabo un análisis secundario, es productivo reflexionar sobre el interés de la investigación y la recopilación de datos de la obra original."

- "Una cuestión importante es qué los temas del proyecto de investigación original coincidan con los del presente proyecto."

- "Si sólo una parte de la muestra son importantes para la investigación de interés actual, tenemos que definir qué elementos de los actuales materiales son de importancia para nosotros. La selección de datos puede basarse en las características (demandado rasgos tales como edad, sexo), contenidos específicos (por ejemplo a través de varias baterías de preguntas) o características de las entrevistas (la hora, el lugar de la entrevista, etc). Es decir, analizar el material del proyecto (ello lleva a considerar a los participantes del mismo tanto investigadores como entrevistadores y el material archivado del mismo)."

- "Además, de la técnica en sí, es preciso examinar ¿qué tipo de enfoque metodológico fue escogido...?"

- Estar alertas respecto a cómo las "nuevas reflexiones se centran en las respuestas y las circunstancias de las entrevistas" originarias" (NOTZ, 2007) 
c) Van den Berg realiza seis recomendaciones para disminuir los obstáculos que producen los "contextos" diferentes de producción de "datos" cuando usamos información secundaria proveniente de entrevistas, ellos son:

“1. Información sobre el contexto discursivo de las respuestas del entrevistado. A menudo las entrevistas de una investigación se graban y desgraban es necesario poseer la grabación a fin de dar cuenta del modo en que se construyó la conversación entre entrevistado y entrevistador.

2. Información acerca de la historia discursiva de las respuestas del entrevistado. Es necesario contar con toda la entrevista, y considerarla como una totalidad.

3. Información sobre las características y antecedentes del entrevistador y entrevistado. Al menos las características sociales que son visibles o cognoscibles para los propios participantes (tales como edad, sexo, etnia, clase social) deben estar disponibles.

4. Información contextual pertinente para la interacción en la realización de la entrevista, tal como las características del lugar, la presencia de terceras personas, etc.

5. Información acerca del modo de selección del entrevistado.

6. Información acerca del contexto social más amplio, no solo de los entrevistados." (VAN DEN BERG, 2005)

Retomando lo afirmado hasta aquí, y más allá de los "pasos" posibles para realizar análisis secundario queremos sostener la importancia de recordar algunas operaciones básicas para hacerlo:

a) M antener una permanente "duda radical" (sensu Bourdieu) sobre lo que para nosotros signifiquen la información re-usada.

b) Establecer un continuo entre la reflexividad de la investigación de origen y la nuestras. 
c) Justificar metodológica, epistemológica y teóricamente las operaciones que realicemos.

d) Evaluar y -en lo posible- hacer visible los contenidos políticos de las elecciones de la investigación de origen y la nuestra.

Si bien, como en toda la metodología de la investigación social, ninguna recomendación debe ser tomada como dogma creemos que las aquí expuestas pueden al menos servir de camino a transitar. En el próximo apartado hacemos algunas propuestas para dicho recorrido.

\section{Algunas propuestas a modo de apertura de un campo de discusion}

Para finalizar el presente trabajo queremos, al menos parcialmente, realizar cuatro tipos de reflexiones en torno al uso de información "secundaria". En primer término queremos re-marcar, desde una perspectiva epistémica, cual es el lugar que puede ocupar el re-uso de la información aludida. En segundo lugar, subrayar tres motivos por los cuales resulta relevante "hacer análisis secundarios". En tercer lugar, queremos proponer como punto de partida de una discusión posterior los rasgos mínimos que todos deberíamos tener presentes para que nuestras propias informaciones puedan ser usadas por otros. Finalmente esbozar algunos de los sentidos posibles que tendría contar en las Ciencias Sociales de América Latina con información para ser re-analizada.

1) Explicar y comprender fenómenos sociales desde una estrategia cualitativa que suponga el análisis secundario implica tematizar a las informaciones disponibles en tanto inscriptas en redes explicativas y constelaciones comprensivas.

Las informaciones recogidas por otros son puntos de referencia de una navegación cuya planificación no ha sido realizada predeterminadamente. Los resultados de entrevistas, historias de vida, etnografías y de 
todo proceso de observación que no hayamos ejecutado como partes integrantes de un "plan" de indagación propio, pueden transformarse muchas veces, en nodos de una red explicativa que mejora nuestra interpretación sobre nuestro propio itinerario.

Los sentidos, significaciones y valoraciones realizadas por otros que anidan en los resultados de sus procesos de captación de información constituyen una constelación de conexiones comprensivas posibles de ser utilizadas en universos observacionales similares. De ahí que dichas constelaciones puedan, bajo las restricciones observacionales adecuadas ser usadas por nosotros para ampliar la comprensión de nuestros propios campos de observación.

Las metáforas de la red explicativa y de las constelaciones compresivas, (tomando a la información disponible, en una como "nodo"; en otra como "conexión") posibilitan, al menos parcialmente, esclarecer el lugar de la información secundaria y su potencial utilización.

2) Cuando se focaliza la discusión en los motivos por los cuales es importante hacer análisis secundarios aparecen, entre otros, tres muy relevantes: a) El análisis de información secundaria permite - dado lo explicado en el punto anterior- hacer observables "estructuras de sentido" que desde la misma investigación que llevamos adelante resultan difícil de ser captadas; b) la re-elaboración de información es una vía para potenciar supuestos no trabajados directamente en nuestra investigación y que muchas veces se tratan como constantes o se dan por sentado, y c) el análisis secundario permite cotejar y robustecer la validez externa de nuestra investigación.

Así se puede entender mejor por qué el análisis secundario no solo es posible sino que hasta deseable, en términos metodológicos, epistémicosy teóricos.

3) Es de una gran relevancia que todos aquellos que hacemos investigación y en especial los que optamos por estrategias cualitativas 
"dejemos en condición" de análisisa las informaciones que construimos. Dicks et Alt. han expuesto una breve lista de puntos a tener en cuenta en las investigaciones cualitativas para "dejarlas en condiciones" de ser re-usadas:

1 - "La naturaleza y la evolución de las relaciones entre investigador y participante, como así también de sus interacciones;

2 - los rasgos epistemológicos, ontológicos y políticos del proyecto, y como estos funcionan en la practica;

3 - los procesos usados para llegar a la interpretación del significado de la información, y el-los tipo(s) de enfoque(s) utilizados en el análisis de datos." (DICKS, B et al. 2006)

Si bien la lista puede ser aumentada y/o corregida creemos que esta es una buena pista para comenzar a repensar la cooperación metodológica y darles sentido a nuestras redes de programas y grupos de investigación.

4) En el contexto de lo arriba expresado creemos conveniente subrayar tal vez algunos puntos muy conocidos por los discursos de las políticas científicas. El análisis de datos cualitativos secundarios trae a la luz la urgencia de potenciar tres elementos en la investigación en nuestro continente: el aumento necesario de la institucionalización de la investigación acompañado por su correlato de calidad en la elaboración y transmisión de información, la necesidad de una articulación creciente entre investigadores, programas y grupos de investigación tendientes a optimizar esfuerzos y, finalmente la urgencia de avanzar en esquemas de cooperación en las investigaciones, mejorando el almacenaje y publicidad de los resultados de las mismas.

En este marco creemos que desde las amplias nociones de datos y análisis secundarios se puede caminar hacia un proceso de re-utilización de información, que implique la creación de una información re-elaborada desde donde aumente las ventajas y disminuyan la desventajas arriba señalas. 
El avance a través de redes explicativas y constelaciones comprensivas construidas de los modos indicados re-potencia la esperanza de lograr cimentar unas Ciencias Sociales que conviertan a los rasgos éticos y contextuales de una investigación, no ya en desventajas del uso de la información, sino en sus propias potencialidades. El otro, los otros y los nosotrosimplicados en los procesos de indagación social se harán más visibles y comprensibles si podemos darles paso a ser vistos y escuchados por una pluralidad de interlocutores.

\title{
The second parts may be better: some reflections on the use of secondary data in qualitative research
}

\begin{abstract}
The analysis of secondary data is one of the "oldest" strategies that sociologists have used to work with the phenomena of social reality when the available information was prepared by others. This study intends to establish a systematic reflection on the use of secondary information in the processes of qualitative social research. The article is divided into four parts: the first works on a definition of data and secondary analysis; the second part discusses advantages and disadvantages of the use of qualitative information; the third asks how, why and what for is the reuse of qualitative information; and finally the fourth part presents some proposals for discussion. The aim is to establish clear criteria that would facilitate the use and analysis of information produced by others and / or in different contexts of production.
\end{abstract}

Keywords: Secondary data. Q ualitative research. Reuse of information.

\section{Referencias}

CEA D 'AN CO NA, M. Ángeles. Metodología cuantitativa: estrategias y técnicas de investigación social. Madrid: Ed. Síntesis sociología, 1998.

CORTI, Louise. Progress and Problems of Preserving and Providing Access to Q ualitative Data for Social Research - The International Picture of an Emerging 
Culture [58 paragraphs]. Forum Q ualitative Sozialforschung / Forum: Q ualitative Social Research [0 n-line Journal], 1(3). December, 2000. Disponível em: < http:/ /www.qualitative-research.net/index.php/fqs/article/view/1019/2198>

DICKS, B., M ASO N, B., W ILLIAMS, M ., \& COFFEY, A. Ethnography and data reuse: issues of context and hypertext. Methodological Innovations O nline [O nline],1(2). December 8, 2006. Disponível em: < http://erdt.plymouth.ac.uk/mionline/ public_html/viewarticle.php?id=28.>

GALTUNG. Johan. Teoría y Método de la Investigación Social. Tomo I y II Bs As: Ed. Eudeba, 1978.

GOODWIN, J., \& O'CONNOR, H.. Contextualising the research process: Using interviewer notes in the secondary analysis of qualitative data. The Q ualitative Report, 11 (2), , 2006. p. 374-392D isponível em: < http://www.nova.edu/ssss/Q R/ QR112/ goodwin.pdf>

HEATON, J. Secondary analysis of qualitative data. Social Research U pdate Issue 22 Autumn UniS University of Surrey, 1998. Disponível em: < http:// sru.soc.surrey.ac.uk/SRU 22.html>

KELD ER, Jo-Anne. Using Someone Else's D ata: Problems, Pragmatics and Provisions [56 paragraphs]. Forum Q ualitative Sozialforschung / Forum: Q ualitative Social Research [0 n-line Journal], 6(1), Art. 39. January, 2005. Disponível em: < http:// www.qualitative-research.net/fqstexte/ 1-05/05-1-39-e.htm>

MAYNTZ, Renate; KART, H ola; HUBNER, M eter. Introducción a los métodos de la sociología empírica. Madrid: Alianza Editorial, 2004.

MRUCK, Katja. Editorial: El volumen FQS sobre "Análisis secundario de datos cualitativos" [6 párrafos]. Forum Q ualitative Sozialforschung/ Forum: Q ualitative Social Research [0n-line Journal], 6(1), Art. 48. Enero, 2005. Disponível em: < http://www.qualitative-research.net/fqstexte/1-05/05-1-48-s.htm>

MO ORE, N. The Contexts of Context: Broadening Perspectives in the (Re)use of Q ualitative Data. Methodological Innovations Online [O nline], 1(2), 2006. Disponível em: < http://erdt.plymouth.ac.uk/mionline/public_html viewarticle. php?id $=27>$

NOTZ, Petra. Secondary Qualitative Analysis of Interviews. A Method Used for Gaining Insight into the Work/Life Balance of Middle Managers in Germany [41 paragraphs]. Forum Q ualitative Sozialforschung / Forum: Q ualitative Social Research, 6(1), Art. 34, 2007. Disponível em: < http://www.qualitative-research.net/ fqs-texte/1-05/05-1-34-e.htm>

PADUA, Jorge. Técnicas de investigación aplicadas a las ciencias sociales. M éxico: Ed. Fondo de Cultura económica, 2000. 
ROJAS SO RIAN O, Raúl. El proceso de la investigación científica. M éxico: Editorial Trillas, 1995.

SAUTU, Ruth; BO NIO LO Paula; DALLE, Pablo; ELBERT, Rodolfo. Manual de metodología. Construcción del marco teórico, formulación de los objetivos y elección de la metodología. Buenos Aires: Colección Campus Virtual. CLACSO libros, 2005.

SCRIBAN O, Adrián. El Proceso de Investigación Social Cualitativo. Pp. 300 Buenos Aires: Editorial Prometeo, 2008.

SELLTIZ, Claire; WRIGHTSMAN, Lawrence; COOK, Stuart. Métodos de investigación en las Relaciones Sociales. Madrid: Ediciones RIALP S.A, 1980.

SIERRA BRAVO, R. Técnicas de Investigación Social. Teoría y ejercicios. Madrid: Thomson Editores, 2003.

VAN DEN BERG, Harry. Reanalyzing Q ualitative Interviews From Different Angles: The Risk of Decontextualization and O ther Problems of Sharing Q ualitative Data [48 paragraphs]. Forum Q ualitative Sozialforschung / Forum: Q ualitative Social Research [O n-line Journal], 6(1), Art. 30. January, 2005. Disponível em: http:// www.qualitative-research.net/fqs-texte/1-05/05-1-30-e.htm

Recebido: 04/02/2009

Aceite final: 02/03/2009 